\title{
Core competencies for patient safety research: a cornerstone for global capacity strengthening
}

\author{
Anne Andermann, ${ }^{1}$ Liane Ginsburg, ${ }^{2}$ Peter Norton, ${ }^{3}$ Narendra Arora, ${ }^{4}$ \\ David Bates, ${ }^{5}$ Albert Wu, ${ }^{6}$ Itziar Larizgoitia, ${ }^{7}$ On behalf of the Patient Safety \\ Research Training and Education Expert Working Group of WHO Patient Safety
}

${ }^{1}$ Woodrow Wilson School of Public and International Affairs, Princeton University, Princeton, New Jersey, USA ${ }^{2}$ School of Health Policy \& Management, York University, Toronto, Canada ${ }^{3}$ Department of Family Medicine, University of Calgary, Ontario, Canada ${ }^{4}$ The INCLEN Trust International, New Delhi, India

${ }^{5}$ Department of Health Policy and Management, Harvard School of Public Health, and Brigham and Women's Hospital, Boston, Massachusetts, USA ${ }^{6}$ Department of Health Policy and Management, Johns Hopkins Bloomberg School of Public Health, Baltimore, Maryland, USA

${ }^{7}$ WHO Patient Safety, World Health Organization, Geneva, Switzerland

Correspondence to Dr Itziar Larizgoitia, WHO Patient Safety, 20 Ave Appia, 1211 Geneva 27,

Switzerland;

Accepted 30 April 2010 larizgoitiai@who.int

Background: Tens of millions of patients worldwide suffer disabling injuries or death every year due to unsafe medical care. Nonetheless, there is a scarcity of research evidence on how to tackle this global health priority. The shortage of trained researchers is a major limitation, particularly in developing and transitional countries. Objectives: As a first step to strengthen capacity in this area, the authors developed a set of internationally agreed core competencies for patient safety research worldwide.

Methods: A multistage process involved developing an initial framework, reviewing the existing literature relating to competencies in patient safety research, conducting a series of consultations with potential end users and international experts in the field from over 35 countries and finally convening a global consensus conference.

Results: An initial draft list of competencies was grouped into three themes: patient safety, research methods and knowledge translation. The competencies were considered by the WHO Patient Safety task force, by potential end users in developing and transitional countries and by international experts in the field to be relevant, comprehensive, clear, easily adaptable to local contexts and useful for training patient safety researchers internationally.

Conclusions: Reducing patient harm worldwide will require long-term sustained efforts to build capacity to enable practical research that addresses local problems and improves patient safety. The first edition of Competencies for Patient Safety Researchers is proposed by WHO Patient Safety as a foundation for strengthening research capacity by guiding the development of training programmes for researchers in the area of patient safety, particularly in developing and transitional countries, where such research is urgently needed.

\section{INTRODUCTION} online under the BMJ Journals unlocked scheme, see http://qualitysafety.bmj. com/site/about/unlocked. xhtml levels of development. WHO Patient Safety (formerly known as the World Alliance for Patient Safety) was established in 2004 to mobilise global efforts to improve the safety of healthcare for patients in all WHO Member States. WHO estimates that millions of patients worldwide suffer disabling injuries or death every year due to unsafe medical practices and care. ${ }^{1}$ While nearly one in ten patients is harmed while receiving healthcare in well-funded and technologically advanced hospital settings, there is little evidence about the burden of unsafe care in developing countries, where the risk of patient harm may be even greater due to limitations in infrastructure, technology and human resources, either in hospital or in primary care and community settings.

WHO Patient Safety gives special emphasis to research advancement as one of the essential building blocks for achieving safer care. Patient safety research is defined as: An actionoriented field of scientific enquiry that aims to determine: 1 ) the type and magnitude of harm caused by unsafe care; 2) the contributing factors and causal pathways that are potentially modifiable, including unsafe systems, processes and behaviours; and 3) cost-effective and locally adapted interventions that can successfully prevent, reduce or mitigate unsafe care to reduce harm. More knowledge - and better use of the knowledge available - are essential for understanding the extent and causes of patient harm, and for developing solutions that can be used in different contexts. To address the lack of research capacity in patient safety research worldwide, especially in developing and transitional countries, WHO Patient Safety convened a task force of world experts in patient safety research, curriculum development and 
research capacity strengthening from a wide range of countries in early 2008. The initial goal was to develop a set of core competencies for patient safety research to guide the development of education and training opportunities for promoting capacity strengthening in this area. Below, we describe the group's approach to competency development, present the competencies, and discuss implications and next steps. The full report is available on the WHO website (http://www.who.int/patientsafety/). ${ }^{2}$

\section{METHODS}

The 21-member international task force convened by WHO Patient Safety followed a seven-stage process (figure 1) to develop patient safety research competencies that would be applicable in developing, transitional and developed countries. The first stage involved preparing a background paper and initial framework for patient safety research competencies. ${ }^{3}$ These documents were discussed at the first meeting of the task force in February 2008 (Stage 2). An expanded literature review of competencies relating to patient safety, research and knowledge translation (Stage 3) was combined with the initial framework from Stage 1 and feedback from Stage 2 to create a preliminary list of patient safety research competencies (version 0.1). An internal consultation with the task force (Stage 4) used an on-line survey to identify key documents or competencies that did not emerge from the Stage 3 literature review, and to suggest changes to the content or format of the preliminary list of competencies. Feedback was incorporated into version 0.2 of the competencies. A large-scale external consultation with potential end users of the competencies (Stage 5) used a snowball technique to send an on-line survey to researchers, practitioners and policymakers in developing and transitional countries working in the area of patient safety, to determine whether the

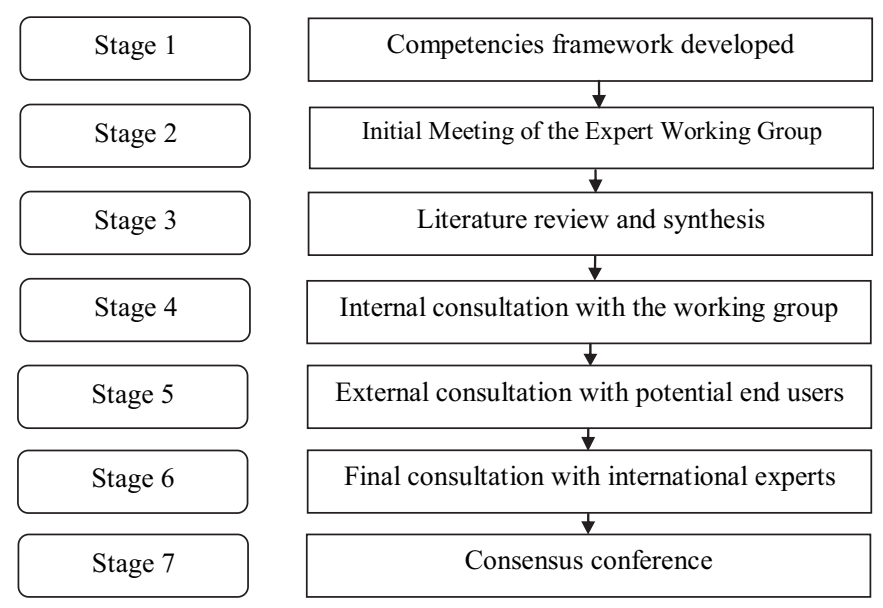

Figure 1 Seven-stage patient safety research competency development process. list of competencies (version 0.2) would be easy to understand, appropriate to local contexts and useful for training future patient safety researchers. Feedback was used to revise the competencies to version 0.3. A second external consultation with international experts in patient safety (Stage 6) involved sending an online questionnaire to a convenience sample of 155 experts chosen from several key meeting and conference lists to assess the face validity of the proposed list of competencies. The sample, which included the external leads of WHO Patient Safety as well as advisory council members for the Research Programme, involved experts from the six WHO world regions, although the predominance was from high-income countries in Europe and North America where patient safety research has been more developed. Version 0.4 of the patient safety research competencies was presented to the task force for discussion at a 2-day conference (Stage 7) aimed at (a) achieving consensus on a first edition of the Patient Safety Research Competencies (version 1.0), as well as (b) identifying steps for further validation and dissemination of the competencies, and for their incorporation into existing or new training programmes in developing and transitional countries in particular.

\section{RESULTS}

In January 2008, when the seven-stage process of competency development began, little had been published on the nature, boundaries and challenges of patient safety research. ${ }^{4-9}$ Since that time, a great deal more has emerged in the literature. ${ }^{10-18}$ The initial framework for patient safety research competencies that emerged from Stage 1 suggested that competencies may differ for different target audiences, different regional contexts and different levels of research advancement (table 1). ${ }^{3}$ The primary discussion points of the international task force (Stage 2) focused on (a) how to develop patient safety research competencies that would be relevant for developing and transitional countries, and (b) who should be the main target audience of patient safety research education and training.

Twenty-nine documents were identified for inclusion in the literature review and synthesis of competencies relating to patient safety research (Stage 3). These included documents on competencies for promoting patient safety, ${ }^{19-22}$ for conducting health services research $^{23}{ }^{24}$ and for knowledge translation. ${ }^{25}{ }^{26} \mathrm{~A}$ preliminary list of patient safety research competencies was created (version 0.1) based on key themes which emerged from the literature (figure 2) relating to patient safety, research, and knowledge translation. Feedback on this preliminary list was obtained from nine out of 21 members (43\%) of the task force (Stage 4). 
Table 1 Initial framework for patient safety research competencies* $^{*}$

\begin{tabular}{|c|c|c|c|}
\hline $\begin{array}{l}\text { Advanced } \\
\text { knowledge and } \\
\text { skills needed in } \\
\text { the following } \\
\text { competency } \\
\text { areas: }\end{array}$ & $\begin{array}{l}\text { Academic } \\
\text { researcher } \\
\text { track }\end{array}$ & $\begin{array}{l}\text { Clinician } \\
\text { researcher } \\
\text { track }\end{array}$ & $\begin{array}{l}\text { Policy/ } \\
\text { manager } \\
\text { researcher } \\
\text { track }\end{array}$ \\
\hline $\begin{array}{l}\text { 1. Science of } \\
\text { patient safety }\end{array}$ & $\sqrt{ }$ & + & $\sqrt{ }$ \\
\hline $\begin{array}{l}\text { 2. At least one } \\
\text { patient safety } \\
\text { subspecialty }\end{array}$ & $\sqrt{ }$ & - & - \\
\hline $\begin{array}{l}\text { 3. Research design } \\
\text { and methodology }\end{array}$ & + & $\sqrt{ }$ & $\sqrt{ }$ \\
\hline $\begin{array}{l}\text { 4. Conducting } \\
\text { research }\end{array}$ & + & $\sqrt{ }$ & $\sqrt{ }$ \\
\hline $\begin{array}{l}\text { 5. Statistics and } \\
\text { data analysis }\end{array}$ & + & $\sqrt{ }$ & $\sqrt{ }$ \\
\hline $\begin{array}{l}\text { 6. Knowledge } \\
\text { translation }\end{array}$ & $\sqrt{ }$ & $\sqrt{ }$ & + \\
\hline
\end{tabular}

Respondents to the internal consultation highlighted the need for further attention to questions about whether and how patient safety research competencies may differ for different researcher profiles (eg, academic researcher, policy-maker, healthcare practitioner interested in research), different regional, social, economic and cultural contexts, and the extent to which the competencies take into account unique contextual issues in developing countries.

In response to these concerns, Stage 5 solicited feedback from potential end users of the competencies in

\section{Patient safety themes}

- able to communicate and work collaboratively to promote continuous quality improvement, institutionalize a culture of patient safety, and encourage the use of standardized protocols, informatics and technology to improve safety, as well as,

- able to emphasize a systems approach to safe and quality care, consider human factors and safety design to prevent patient safety incidents, as well as handle patient safetyrelated incidents in a timely and open manner.

Research themes

- able to understand and apply research methods, conduct ethical research that answers important questions and contributes to the body of knowledge on patient safety

- able to conduct literature reviews, formulate sound research questions, design research protocols, engage in data collection, analysis, interpretation, and dissemination activities to communicate research findings and translate them into concrete proposals for action or change, as well as,

- proficient in computer literacy, grant writing and management of the logistics of the research process, as well as teaching and mentoring other researchers.

Knowledge translation themes

- able to utilize research findings as a basis for improving patient safety through the adoption, implementation and institutionalization of practices and policies that contribute to safer care, as well as,

- able to find, appraise and synthesize the evidence, to translate research findings into concrete changes, to communicate effectively to a variety of audiences, to employ change management techniques, and to take a leadership role in promoting patient safety within an organization

Figure 2 Emerging themes relating to patient safety research competencies. developing and transitional countries. The external consultation yielded usable data from 73 respondents in 35 developing and transitional countries across all six WHO regions. Respondents were largely early to midcareer academics or physicians involved in conducting research relating to patient safety $(96 \%)$, with one-third focussing on safe medicines and devices, and another third focussing on healthcare associated infections. Respondents indicated that the proposed patient safety research competencies were easy to understand and relevant in developing and transitional countries, and would be useful for training patient safety researchers in these regions. Respondents also indicated that few patient safety research training opportunities presently exist (see table 2). At least half of respondents believed that each of the three competency areas is important for practitioners, policy-makers and academic researchers alike, thus indicating that a single list of competencies for all three profiles of patient safety researchers should be retained in version 0.3 .

Of the 46 international experts who responded in Stage $6,85 \%$ found the competencies easy to understand, $87 \%$ considered them to be appropriate for local contexts, and $93 \%$ thought they would be helpful for training future patient safety researchers internationally (see table 2). They also emphasised the need to avoid the use of technical jargon (such as 'disseminating' and 'surveillance') and the need for subdividing competencies that cover multiple concepts and grouping together those where there is overlap.

In the final stage of the competency development process (Stage 7), 17 of the 21 members of the task force, as well as three key informants, attended a consensus conference for an in-depth discussion of version 0.4 of the patient safety research competencies. During the conference, modifications were made to better reflect that the main target audience are researchers (as opposed to end users of research such as clinicians or policy-makers) and to ensure that the final document would employ appropriate terminology commonly used in the field of competency development. Consensus was reached that since all competencies contained basic and advanced levels, there was no need to specify competencies as basic or advanced for trainees at different academic levels. The first edition of the Competencies for Patient Safety Research (version 1.0-box 1) was agreed upon at the close of the meeting.

\section{DISCUSSION}

Patient safety research competencies represent the fundamental knowledge, ability, skills and expertise needed to carry out research in this area and to use 
Table 2 External consultation key results

\section{Potential end users from developing and transitional countries (Stage 5 respondents, $n=73$ )}

International experts in patient safety research (Stage 6 respondents, $n=46$ )

Percentage reporting the competencies

Are easy to understand

$63(86 \%)$

$60(82 \%)$

$64(88 \%)$

$73(100 \%)$
$39(85 \%)$

$32(70 \%)$

$40(87 \%)$

$43(93 \%)$

Would be useful for training patient safety researchers

Percentage reporting the competencies would be useful As a systematic basis for training $60(82 \%)$

For defining learning objectives $55(75 \%)$

To emphasise different knowledge $\quad 53(73 \%)$

$37(80 \%)$

$33(72 \%)$

$37(80 \%)$

and skills needed

As a basis to be tailored to different

trainee profiles

To evaluate the progress of trainees

$37(51 \%)$

$25(54 \%)$

$57(78 \%)$

$23(50 \%)$

Competency area considered the main priority for training patient safety researchers in their own country:

Patient safety theory and practice

$31(42 \%)$

NA

Designing and conducting research

$24(33 \%)$

Translating findings into safer care

$18(25 \%)$

Percentage aware of training

$13(18 \%)$

NA

researchers in their country

patient safety research evidence to make healthcare safer. The current paper describes the methods and results of a multistage process, including literature reviews and consultations with experts and stakeholders that were used to develop patient safety research competencies. The competencies that emerged are intended to guide patient safety researchers in acquiring the knowledge and skills needed to conduct research that aims to better understand the magnitude and type of patient harm, identify potentially modifiable contributing factors, and design and test cost-effective and locally adapted interventions that can successfully prevent, reduce or mitigate unsafe care to reduce harm.

The first edition of Competencies for Patient Safety Research (version 1.0-box 1) proposed in this paper emphasises (a) understanding the science of patient safety, (b) conducting valid and ethical research and (c) translating research into practice. Our results indicate that both experts and potential end users believe the competencies can be tailored to different audiences, local and national contexts, and levels of educational attainment. Our results further suggest the core competencies have potential to be used by educational and research institutions in high- as well as low- and middle-income country contexts to inform curricula and the development of training programmes. In particular, the core competencies can be used to (a) provide a systematic basis for training, (b) define key learning objectives that should be covered, (c) demonstrate the many different types of knowledge and skills that are needed to carry out patient safety research, (d) tailor training programmes and curricula to different professional profiles, contexts and needs, and (e) help evaluate the progress of patient safety research trainees.

Experience utilising the proposed patient safety research competencies will be essential to achieve a better understanding of the completeness, specificity and acceptability by different user groups in different socio-economic environments, as well as the feasibility of incorporating the competencies into a variety of educational programmes and training modalities. This will require the more general competency areas of 'designing and conducting patient safety research' and 'translating research evidence to improve the safe care of patients' to be adapted to the field of patient safety research by drawing upon context-specific examples, case studies, data collection tools and study designs that are particularly useful in trying to address research questions relating to patient safety. ${ }^{27}$

\section{Implications for policy and practice}

Relatively little is known about the epidemiology of patient safety in developing or transitional countries, and about what strategies will be effective in improving 
Box 1 First edition of Core Competencies for Patient Safety Research

Patient safety researchers are able to:

1. Describe the fundamental concepts of the science of patient safety, in their specific social, cultural and economic context.

These concepts include the following items, among others:

1.1. Basic definitions and foundational concepts, including human factors and organisational theory

1.2. The burden of unsafe care

1.3. The importance of a culture of safety

1.4. The importance of effective communication and collaboration in care delivery teams

1.5. The use of evidence-based strategies for improving the quality and safety of care

1.6. The identification and management of hazards and risks

1.7. The importance of creating environments for safe care

1.8. The importance of educating and empowering patients to be partners for safer care

2. Design and conduct patient safety research. These competencies include the ability to perform, but are not necessarily restricted to, the following:

2.1. Search, appraise and synthesise the existing research evidence

2.2. Involve patients and carers in the research process starting with defining the research objectives

2.3. Identify research questions that address important knowledge gaps

2.4. Select an appropriate qualitative or quantitative study design to answer the research question

2.5. Conduct research using a systematic approach, valid methodologies and information technology

2.6. Employ valid and reliable data measurement and data analysis techniques

2.7. Foster interdisciplinary research teams and supportive environments for research

2.8. Write a grant proposal

2.9. Obtain research funding

2.10. Manage research projects

2.11. Write-up research findings and disseminate key messages

2.12. Evaluate the impact of interventions as well as feasibility and resource requirements

2.13. Identify and evaluate indicators of patient safety for use in monitoring and surveillance

2.14. Ensure professionalism and ethical conduct in research

3. Be part of the process of translating research evidence to improve the safe care of patients. The skills involved include, but are not restricted to, the ability to contribute to the following:

3.1. Appraise and adapt research evidence to specific social, cultural and economic contexts

3.2. Use research evidence to advocate for patient safety

3.3. Define goals and priorities for making healthcare safer

3.4. Translate research evidence into policies and practices that reduce harm

3.5. Partner with key stakeholders in overcoming barriers to change

3.6. Promote standards and legal frameworks to improve safety

3.7. Institutionalise changes to build supportive systems for safer care

3.8. Apply financial information for knowledge translation

3.9. Promote leadership, teaching and safety skills.

patient safety in these regions. Further research is therefore needed, which will require the creation of a critical mass of newly trained researchers able to fill the many knowledge gaps that exist. For many, the goal of education and training in patient safety research is to create research that leads to actions that improve patient safety. However, the link between training patient safety researchers and these more distal outcomes will likely be difficult to demonstrate in the short-term. Accordingly, process indicators such as increases in the number of courses offered in patient safety research, the number of graduates, the local adoption of evidence-based safe practices or the number of policies aimed at improving safety would also be important measures of success.

Building research capacity is a long-term process that requires sustained effort, in terms of formal training opportunities but, more importantly, in providing a nurturing environment for trainees to continue to develop their knowledge and skills by being involved in conducting research. The Core Competencies for Patient Safety Research provide a framework for the ongoing education and training of patient safety researchers worldwide. Establishing formal training programmes at accredited academic institutions across all WHO regions may take many years. However, the growth of research networks and the availability of targeted funding to support patient safety research can already be used to promote research-capacity strengthening, particularly in developing and transitional countries where such research is urgently needed.

Acknowledgements The authors would like to thank the members of the WHO Patient Safety Research Training and Education Expert Working Group for their important contribution to this work: Ahmed Al-Mandhari (Sultan Qaboos University Hospital, Sultanate of Oman); Antoine Geissbuhler (Hôpitaux Universitaires de Genève, Switzerland); John Goshbee (University of Michigan, USA); Festus Ilako (African Medical and Research Foundation, AMREF, Kenya); Mark Joshi (University of Nairobi, Kenya); Rakesh Lodha 
(All India Institute of Medical Sciences, India); Sergio Muñoz Navarro (Universidad de La Frontera, Chile); Hillegonda Maria Dutilh Novaes (University of São Paolo, Brazil); John Orav (Harvard School of Public Health, USA); Alan Pearson (Johanna Briggs Institute, Australia); Peter Pronovost (Johns Hopkins School of Medicine, USA); Miguel Recio Segoviano (Universidad Carlos III de Madrid, Spain); Jiruth Sriratanaban (Chulalongkorn University, Thailand); Naruo Uehara (Tohoku University, Japan); Steven Wayling (Special Programme for Research and Training in Tropical Diseases, World Health Organization, Switzerland); Maria Woloshynowych (Imperial College, United Kingdom); and Zhao Yue (Peking University People's Hospital, P.R. China). The authors would also like to thank Jason Frank (Royal College of Physicians and Surgeons of Canada, Canada) for sharing his expertise in the area of competency development, as well as the many interns who contributed at various stages to this project: Khalifa Elmusharaf (University of Khartoum, Sudan); Aimee McHale (University of North Carolina, USA); Jean-Louis Keene (McGill University, Canada); Naomi Dove (University of British Columbia, Canada); Ruramayi Rukini (University of Bristol, United Kingdom) and Shannon Gibson (University of Victoria, Canada). The authors are also grateful to all the experts and stakeholders from around the world who provided feedback on earlier drafts of this work.

Competing interests None.

Provenance and peer review Not commissioned; externally peer reviewed.

\section{REFERENCES}

1. The Research Priority Setting Working Group of the WHO World Alliance for Patient Safety. Summary of the evidence on patient safety: implications for research. Geneva: World Health Organization, 2008. http://whqlibdoc.who.int/publications/2008/ 9789241596541 eng.pdf

2. The Patient Safety Research Training and Education Expert Working Group of WHO. Patient Safety. Competencies for Patient Safety Research. Geneva: World Health Organization, 2010. http://www. who.int/patientsafety/research.

3. Ginsburg LR, Norton PG. Background paper 1: creating leaders in patient safety research: Essential competencies for conducting research and translating evidence to reduce patient harm. Prepared for the 1st meeting of the Patient Safety Research Training and Education Expert Working Group of WHO Patient Safety, 18-19 February 2008, WHO, Geneva, Switzerland.

4. Meyer GS, Battles J, Hart JC, et al. The US agency for healthcare research and quality's activities in patient safety research. Int J Qual Health Care 2003;15(Suppl 1):25-30.

5. Battles JB, Lilford RJ. Organizing patient safety research to identify risks and hazards. Qual Saf Health Care 2003;12(Suppl 2):2-7.

6. Lilford RJ. Patient safety research: does it have legs? Qual Saf Health Care 2002;11:113-14

7. Meyer GS, Eisenberg JM. The end of the beginning: the strategic approach to patient safety research. Qual Saf Health Care 2002;11:3-4
8. Grol R, Berwick DM, Wensing M. On the trail of quality and safety in health care. BMJ 2008;336:74-6.

9. Cook RL. Lessons from the war on cancer: the need for basic research on safety. J Patient Saf 2005;1:7-8.

10. Brown $\mathrm{C}$, Hofer $\mathrm{T}$, Johal $\mathrm{A}$, et al. An epistemology of patient safety research: a framework for study design and interpretation. Part 1. Conceptualising and developing interventions. Qual Saf Health Care 2008;17:158-62.

11. Brown C, Hofer $\mathrm{T}$, Johal $\mathrm{A}$, et al. An epistemology of patient safety research: a framework for study design and interpretation. Part 2. Study design. Qual Saf Health Care 2008;17:163-9.

12. Brown $\mathrm{C}$, Hofer $\mathrm{T}$, Johal $\mathrm{A}$, et al. An epistemology of patient safety research: a framework for study design and interpretation. Part 3. End points and measurement. Qual Saf Health Care 2008;17:170-7.

13. Brown C, Hofer T, Johal A, et al. An epistemology of patient safety research: a framework for study design and interpretation. Part 4. One size does not fit all. Qual Saf Health Care 2008;17:178-81.

14. Bates DW. Mountains in the clouds: patient safety research. Qual Saf Health Care 2008;17:156-7.

15. Kass N, Pronovost PJ, Sugarman J, et al. Controversy and quality improvement: lingering questions about ethics, oversight, and patient safety research. Jt Comm J Qual Patient Saf 2008;34:349-53.

16. Hofoss $D$, Deilkås $E$. Roadmap for patient safety research: approaches and roadforks. Scand J Public Health 2008;36:812-17.

17. Pronovost PJ, Goeschel CA, Marsteller JA, et al. Framework for patient safety research and improvement. Circulation 2009;119:330-7.

18. McCarthy $M$, Brookes $M$, Scourfield $P$. Patient safety research: shaping the European agenda. Clin Med 2009;9:145-6.

19. Cronenwett L, Sherwood G, Barnsteiner J, et al. Quality and safety education for nurses. Nurs Outlook 2007;55:122-31.

20. Sandars J, Bax N, Mayer D, et al. Educating undergraduate medica students about patient safety: priority areas for curriculum development. Med Teach 2007;29:60-1.

21. Singh R, Naughton B, Taylor JS, et al. A comprehensive collaborative patient safety residency curriculum to address the ACGME core competencies. Med Educ 2005;39:1195-204.

22. Walton M. The National Patient Safety Education Framework. Sydney, Australia: The Australian Council for Safety and Quality in Health Care and The University of Sydney, 2005.

23. AHRQ. Health Services Research Core Competencies. Final Report Rockville, MD: Agency for Healthcare Research and Quality, 2007.

24. Burke L, Schlenk E, Sereika S, et al. Developing research competence to support evidence-based practice. J Prof Nurs 2005;21:358-63.

25. Armstrong $\mathrm{R}$, Waters $\mathrm{E}$, Roberts $\mathrm{H}$, et al. The role and theoretical evolution of knowledge translation and exchange in public health. J Public Health 2006;28:384-9.

26. Jacobson N, Butterill D, Goering P. Development of a framework for knowledge transfer: understanding user context. $J$ Health Serv Res Policy 2005;8:94-9.

27. WHO Patient Safety. Case Studies in Patient Safety Research. Geneva: World Health Organization, 2009. http://www.who.int/ patientsafety/research/strengthening_capacity/classics/. 